Gynaecologia 1959;149(Suppl. 1):I-IV

\title{
Contents, Vol. 149, Supplement 1, 1959
}

\section{INDEX}

Part Three Troisième partie Dritter Teil

Chapter i Chapitre i Kapitel i

Main Lecture Conference magistrate Hauptreferat

Chappaz, G. (Reims):

Étiologie de la trichomonase feminine - Etiology of Female Tri-chomoniasis - Ätiolo $7 / 8$ ie der weiblichen Trichomonasinfektion ..

Chapter ii Chapitre ii Kapitel h

Free Communications Communications libres Freie Vorträge

Inoki, S.; Nakanishi, K. and Nakabayashi, T. (Osaka):

Electron Microscopic Observations of Trichomonas vaginalis Em ploying the Thin-Section Technique - Etude du trichomonas vagi nalis au microscope électronique par la technique des coupes minces Elektronenmikroskopische Beobachtungen von Trichomonas vagi nalis bei der Anwendung der dünnen Schnittechnik ....... 48 Lang, W. R.; Fritz, M. A. and Menduke, H. (Philadelphia, Pa.): Bacterial and Mycotic Components of Trichomonas vaginalis vagi nitis - La flore bactérienne et mycosíque dans les vaginites à tricho monas vaginalis - Bakterielle und mykotischc Komponenten bei der Trichomonas vaginitis $\quad 55$

McEwen, D. C. (Regina, Sask.):

Common Factors in Trichomonas vaginitis - Les facteurs communs de la vaginite à trichomonas - Allgemeine Gesichtspunkte über die Trichomonas vaginitis

63

Kostic, P. (Belgrade):

Le trichomonas vaginal du tractus urinaire chez la femme -

Trichomonas vaginalis in the Female Urinary Tract - Die Vaginaltrichomonas im Harnweg der Frau 70

Youngblood, V. H.; Tomlin, E. M. and Williams, J. O. (Concord, N.C): Senile Urethritis in Women - L'urétrite senile chez la femme - Die Altersurethritis bei der Frau $\quad 76$

$\mathrm{m}$

Heltai, A. (New York, N. Y.):

Hemophilus vaginalis et les vaginites non spécifîques - Hcmophilus vaginalis and Nonspecific Vaginitis - Haemophilias vaginalis und unspezifische Vaginitis 80

Catterall, R. D. and Nicol, C. S. (Leeds): 
Is Trichomonal Infestation a Venereal Disease? - L'infestation à trichomonas est-elle une maladie vénérienne ? - 1st die Trichomonas eine venerische Erkrankung? 87

Bertrand, P. et Leulier, J. (Reims):

Essais cliniques sur la trichomonase des partenaires des femmes infestées - Clinical Examinations of the Partners of Women with Trichomonas - Klinische Untersuchungen über die Trichomonas bei Partnern von infizierten Frauen 93

Kean, B. H. (New York, N. Y,):

Conjugal Trichomoniasis - La trichomonase conjugale - Die eheliche Trichomonas 97

Part Four Quatrième partie Vierter Teil Chapter i Crapitre i Kapitel i

Main Lecture Conference magistrate Hauptreferat

Feo, L. G. (Philadelphia, Pa.):

Treatment of Trichomoniasis in the Female - Traitement de la tri chomonase feminine - Behandlung der Trichomonasinfektion bei der Frau 101

Chapter ii Chapitre ii Kapitel $\pi$

Free Communications Communications libres Freie Vorträge

Kupferberg, A. B. (Raritan, N.J.):

Metabolic Studies and Chemotherapy of Trichomonas vaginalis -Etudes métaboliques et chimiothérapie du trichomonas vaginalis -StofFwechselstudien und Chcmotherapie von Trichomonas vaginalis 114

Willcox, R. R. (London):

Treatment of Vaginal Trichomoniasis with Aminitrozole and Trichomycin Given Orally - Traitement de la trichomonase vaginale par l'Aminitrozole et la Trichomycine par voie buccale - Behandlung der Vaginaltrichomonas mit Aminitrozol und Trichomycin durch orale Applikation 122

IV Jones, C. P.; Carter, B. and Thomas, W. L. (Durham, N.C.):

The Treatment of Resistant or Recurrent Vaginal Trichomoniasis with Lactic Acid Jelly and Lactic Acid Douches - Traitement des trichomonases vaginales rebelles ou récidivantes par la gelée et les injections vaginales d'acide lactique - Die Behandlung von resistenter oder rückfälliger Vaginaltrichomonas mit Milchsäuregelee und Milchsäureduschen 128

Siboulet, A. (Paris):

A propos du traitement de la trichomonase uro-génitale feminine The Treatment of the Urogenital Trichomonas Infection in the Female - Uber die Behandlung der weiblichen Urogenítaltrichomonasinfektion 139

Magara, M.; Nakamura, J.; Amino, E. and Nittono, F. (Tokyo): Therapeutic Effect of Antitrichomonad Drugs in Female Tricho moniasis - Action thérapeutique des produits antitrichomonasiques 
dans la trichomonase feminine - Die therapeutische Wirkung von Antitrichomonasemedikamenten bei der Frau 143

Bedoya, J. M. (Seville):

"The Multiple Local Treatment" of Human Genital Trichomoniasis

- «Le traitement local multiple» dans la trichomonase génitale

humaine - Die «multiple lokale Behandlung» der menschlichen

Genitaltrichomonas 148

Thiery, M.; Onghena, G.; Caspary, J. et Waefelaer, A. (Gand):

Traitement de la vaginite à trichomonas à $\Gamma$ aide de derives de

ГAmino-2, Nitro-5, Thiazol - Treatment of Trichomonas vaginitis

with Derivatives of 2-Amino-5-Nitrothiazol - Behandlung von

Trichomonas vaginitis mit Derivaten von Amino-2 Nitro-5 Thia

zol 154

Fortier, L. (Montreal):

Traitement de la trichomonase chez la femme par un nouveau derive de Cimidazole - Treatment of Trichomoniasis in the Female with a New Imidazole Derivative - Behandlung der Trichomonas der Frau mit einem neuen Imidazolderivat 158

Bibliography of Parts Three and Four - Bibliograpiiie des 3ème et 4ème parties Literatur zum dritten und vierten Teil 165

Resolution - Resolution - Beschluss 171 